\title{
Localized Majorana-Like Modes in a Number-Conserving Setting: An Exactly Solvable Model
}

\author{
Fernando Iemini, ${ }^{1,2}$ Leonardo Mazza, ${ }^{2}$ Davide Rossini, ${ }^{2}$ Rosario Fazio, ${ }^{2,4}$ and Sebastian Diehl ${ }^{3}$ \\ ${ }^{1}$ Departamento de Física-ICEx-Universidade Federal de Minas Gerais, Belo Horizonte, Minas Gerais, Brazil \\ ${ }^{2}$ NEST, Scuola Normale Superiore \& Istituto Nanoscienze-CNR, I-56126 Pisa, Italy \\ ${ }^{3}$ Institute of Theoretical Physics, TU Dresden, D-01062 Dresden, Germany \\ ${ }^{4}$ ICTP, Strada Costiera 11, 34151 Trieste, Italy
}

(Received 20 April 2015; published 7 October 2015)

\begin{abstract}
In this Letter we present, in a number conserving framework, a model of interacting fermions in a twowire geometry supporting nonlocal zero-energy Majorana-like edge excitations. The model has an exactly solvable line, on varying the density of fermions, described by a topologically nontrivial ground state wave function. Away from the exactly solvable line we study the system by means of the numerical density matrix renormalization group. We characterize its topological properties through the explicit calculation of a degenerate entanglement spectrum and of the braiding operators which are exponentially localized at the edges. Furthermore, we establish the presence of a gap in its single particle spectrum while the Hamiltonian is gapless, and compute the correlations between the edge modes as well as the superfluid correlations. The topological phase covers a sizable portion of the phase diagram, the solvable line being one of its boundaries.
\end{abstract}

PACS numbers: 71.10.Pm, 03.65.Vf, 03.75.Ss, 74.78.-w

Introduction.-A large part of the enormous attention devoted in the last years to topological superconductors is due to the exotic quasiparticles such as Majorana modes, which localize at their boundaries (edges, vortices, ...) [1,2] and play a key role in several robust quantum information protocols [3]. Kitaev's $p$-wave superconducting quantum wire [4] provides a minimal setting showcasing all the key aspects of topological states of matter in fermionic systems. The existence of a so-called "sweet point" supporting an exact and easy-to-handle analytical solution puts this model at the heart of our understanding of systems supporting Majorana modes. Various implementations in solid state $[5,6]$ and ultracold atoms [7,8] via proximity to superconducting or superfluid reservoirs have been proposed, and experimental signatures of edge modes were reported [9].

Kitaev's model is an effective mean-field model and its Hamiltonian does not commute with the particle number operator. Considerable activity has been devoted to understanding models supporting Majorana edge modes in a number-conserving setting [10-14], as in various experimental platforms (e.g., solid state $[10,11]$ or ultracold atoms $[12,13])$ this property is naturally present. It was realized that a simple way to promote particle number conservation to a symmetry of the model, while keeping the edge state physics intact, was to consider at least two coupled wires rather than a single one [10-12]. However, since attractive interactions are pivotal to generate superconducting order in the canonical ensemble, one usually faces a complex interacting many-body problem. Therefore, approximations such as bosonization [10-12], or numerical approaches [13] were invoked. An exactly solvable model of a topological superconductor in a number conserving setting, which would directly complement Kitaev's scenario, is missing (see however Ref. [14]).

In this Letter we present an exactly solvable model of a topological superconductor which supports exotic Majorana-like quasiparticles at its ends and retains the fermionic number as a well-defined quantum number. The construction of the Hamiltonian with local two-body interactions and of its ground state draws inspiration from ideas on dissipative state preparation for ultracold atomic fermions [15-17], here applied to spinless fermions in a two-wire geometry. The solution entails explicit ground state wave functions, which feature all the main qualitative properties highlighted so far in approximate analytical $[12,18,19]$ and numerical $[13,19]$ studies for this class of models, with the advantage of being easy to handle.

In particular, we establish the following key features: (i) the existence of one (two) degenerate ground states depending on the periodic (open) boundaries with a twofold degenerate entanglement spectrum; (ii) the presence of exponentially localized, symmetry-protected edge states and braiding matrices associated to this degeneracy; (iii) exponential decay of the fermionic single particle correlations, even if the Hamiltonian is gapless with collective, quadratically dispersing bosonic modes; (iv) $p$-wave superconducting correlations which saturate at large distance.

By tuning the ratio of interaction vs kinetic energy of our model, we can explore its properties outside the exactly solvable line. The full phase diagram (Fig. 1) is obtained by means of density matrix renormalization group (DMRG) calculations. The exactly solvable line is found to stand 


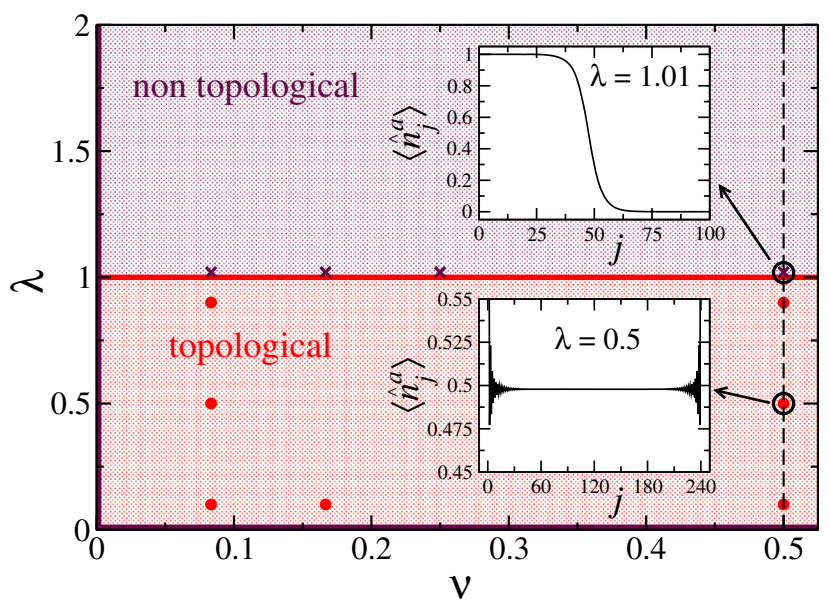

FIG. 1 (color online). Phase diagram for the number and local parity conserving two-wire model [Eq. (3)] as a function of $\lambda$ and filling $\nu=N / 2 L$ obtained through DRMG simulations. The exactly solvable topological line is at $\lambda=1$ (another, trivially solvable nontopological line is at $\lambda=0$ ). For $\lambda>1$, the system undergoes phase separation (see the density profile $\left\langle\hat{n}_{j}^{a}\right\rangle$ in the inset). For $0<\lambda<1$ and $\nu \neq 0,1$, the system is in a homogeneous topological phase (see inset). The phase diagram is symmetric with respect to half filling $\nu=1 / 2$ due to particle-hole symmetry of $\hat{H}_{\lambda}$.

between a stable topological phase and a phaseseparated state.

The model.-We begin by recapitulating some properties of the Kitaev chain, whose Hamiltonian reads [4]

$$
\hat{H}_{K}=\sum_{j}\left[-J \hat{a}_{j}^{\dagger} \hat{a}_{j+1}-\Delta \hat{a}_{j} \hat{a}_{j+1}+\text { H.c. }-\mu\left(\hat{n}_{j}-1 / 2\right)\right] .
$$

Here, $J>0$ denotes the hopping amplitude, $\mu$ and $\Delta$ the chemical potential and the superconducting gap, respectively; $\hat{a}_{j}^{(\dagger)}$ are fermionic annihilation (creation) operators on site $j$, and $\hat{n}_{j} \equiv \hat{a}_{j}^{\dagger} \hat{a}_{j}$. This model has (i) two densitydriven phase transitions from finite densities to the empty and full states at $|\mu|=2 J$ (for $\Delta \neq 0$ ), and (ii) a transition driven by the competition of kinetic and interaction energy (responsible for pairing) at $\Delta / J=0$ (for $|\mu|<2 J$ ). For $|\mu|<2 J$ and $\Delta \neq 0$, the ground state is unique for periodic boundary conditions, but twofold degenerate for open geometry, hosting localized zero-energy Majorana modes. This topological phase is symmetry protected by total fermionic parity $\hat{P}=(-1)^{\hat{N}}$, where $\hat{N} \equiv \sum_{j} \hat{n}_{j}$.

Let us focus on the so-called "sweet point," namely, $\mu=0$, and $\Delta=J>0$ and real, which enjoys the property $\hat{H}_{K}=(J / 2) \sum_{j} \hat{\ell}_{j}^{\dagger} \hat{\ell}_{j}$ with $\hat{\ell}_{j}=\hat{C}_{j}^{\dagger}+\hat{A}_{j}, \hat{C}_{j}^{\dagger}=\hat{a}_{j}^{\dagger}+\hat{a}_{j+1}^{\dagger}$ and $\hat{A}_{j}=\hat{a}_{j}-\hat{a}_{j+1}\left(\hat{\ell}_{L}\right.$ is defined identifying $\left.L+1 \equiv 1\right)$. For open geometry, the two ground states with $L$ sites satisfy $\hat{\ell}_{j}|\psi\rangle=0$, for $1 \leq j \leq L-1$, and can be written [20] as the equal weighted superposition of all even $(e)$ or odd $(o)$ particle number states:

$$
|\psi\rangle_{e(o)}=\mathcal{N}_{e(o), L}^{-1 / 2} \sum_{n}(-1)^{n} \sum_{\left\{\vec{j}_{2 n(2 n+1)}\right\}}\left|\vec{j}_{2 n(2 n+1)}\right\rangle .
$$

Here $\left|\vec{j}_{m}\right\rangle=\hat{a}_{j_{1}}^{\dagger} \hat{a}_{j_{2}}^{\dagger} \ldots \hat{a}_{j_{m}}^{\dagger}|\operatorname{vac}\rangle$ with $j_{i}<j_{i+1}\left(j_{i}=1, \ldots, L\right)$ and $\mathcal{N}_{e, L}=\sum_{n}\left(\begin{array}{c}L \\ 2 n\end{array}\right) ; \mathcal{N}_{o, L}=\sum_{n}\left(\begin{array}{c}L \\ 2 n+1\end{array}\right)$.

We now turn to a number conserving version of this model on a single wire [16]. Indeed, the following model reduces precisely to the above scenario upon performing a naive BCS mean field treatment. Consider the Hamiltonian $\hat{H}_{K}^{\prime} \equiv \sum_{j} \hat{L}_{j}^{\dagger} \hat{L}_{j}$, with $\hat{L}_{j}=\hat{C}_{j}^{\dagger} \hat{A}_{j}$, whose exact ground state wave functions can be obtained as follows. Since $\hat{A}_{j}|\psi\rangle_{e(o)}=-\hat{C}_{j}^{\dagger}|\psi\rangle_{e(o)},|\psi\rangle_{e(o)}$ are also ground states of $\hat{H}_{K}^{\prime}: \hat{L}_{i}|\psi\rangle_{e(o)}=0$ because $\left(\hat{C}_{j}^{\dagger}\right)^{2}=0$. As $\hat{L}_{i}$ conserves the particle number, $\left[\hat{L}_{i}, \hat{N}\right]=0$, we can classify ground states for each fixed particle number sector $N$ by number projection, $|\Psi, N\rangle=\hat{P}_{N}|\psi\rangle_{e(o)}$. This is implemented by choosing the state with $2 n=N$ (or $2 n+1=N$ ) in the sum over $n$ in Eq. (1), and adjusting the normalization to $\mathcal{N}_{L, N}=\left(\begin{array}{l}L \\ N\end{array}\right)$. The positive semidefiniteness of $\hat{H}_{K}^{\prime}$ implies that these states, having zero energy eigenvalue, are ground states. However, once $N$ is fixed, the ground state $|\Psi, N\rangle$ is unique, as follows from the Jordan-Wigner mapping to the Heisenberg model [21]. The topological twofold degeneracy is lost.

Guided by the previous analysis, we construct an exactly solvable topological two-wire model with fermionic operators $\hat{a}_{j}^{(\dagger)}, \hat{b}_{j}^{(\dagger)}$. In addition to those involving each wire $\hat{L}_{a(b), j}=\hat{C}_{a(b), j}^{\dagger} \hat{A}_{a(b), j}$, we introduce new operators $\hat{L}_{I, j}=$ $\hat{C}_{a, j}^{\dagger} \hat{A}_{b, j}+\hat{C}_{b, j}^{\dagger} \hat{A}_{a, j}$. The Hamiltonian

$$
\hat{H}=\sum_{\alpha=a, b, I} \sum_{j=1}^{L-1} \hat{L}_{\alpha, j}^{\dagger} \hat{L}_{\alpha, j}
$$

coincides with the $\lambda=1$ point of the following more general model:

$$
\begin{aligned}
\hat{H}_{\lambda}= & -4 \sum_{j=1, \alpha=a, b}^{L-1}\left[\left(\hat{\alpha}_{j}^{\dagger} \hat{\alpha}_{j+1}+\text { H.c. }\right)-\left(\hat{n}_{j}^{\alpha}+\hat{n}_{j+1}^{\alpha}\right)+\lambda \hat{n}_{j}^{\alpha} \hat{n}_{j+1}^{\alpha}\right] \\
& -2 \lambda \sum_{j=1}^{L-1}\left[\left(\hat{n}_{j}^{a}+\hat{n}_{j+1}^{a}\right)\left(\hat{n}_{j}^{b}+\hat{n}_{j+1}^{b}\right)-\left(\hat{a}_{j}^{\dagger} \hat{a}_{j+1} \hat{b}_{j}^{\dagger} \hat{b}_{j+1}\right.\right. \\
& \left.\left.+\hat{a}_{j}^{\dagger} \hat{a}_{j+1} b_{j+1}^{\dagger} \hat{b}_{j}-2 \hat{b}_{j}^{\dagger} \hat{b}_{j+1}^{\dagger} \hat{a}_{j+1} \hat{a}_{j}+\text { H.c. }\right)\right] .
\end{aligned}
$$

$\hat{H}_{\lambda}$ conserves the total particle number $\hat{N}=\hat{N}_{a}+\hat{N}_{b}$ and the local wire parities $\hat{P}_{a, b}=(-1)^{\hat{N}_{a, b}}$, which act as protecting symmetries for the topological phase. The coupling $\lambda$ tunes the relative strength of the kinetic and interaction terms similarly to $\Delta / J$ in $\hat{H}_{K}$. Although only $\lambda=1$ is exactly solvable, we will later consider $\lambda \neq 1$ to explore the robustness of the analytical results. The phase diagram is anticipated in Fig. 1. 
Exact results for $\lambda=1$.-For a fixed particle number $N$ and open boundaries, the ground state of $\hat{H}$ is twofold degenerate, due to the freedom in choosing the local parity. For even $N$, the ground states read

$$
\begin{aligned}
\left|\psi_{L}(N)\right\rangle_{e e} & =\mathcal{N}_{e e, L, N}^{-1 / 2} \sum_{n=0}^{N / 2} \sum_{\substack{\left\{\vec{j}_{2 n}\right\},\left\{\bar{q}_{N-2 n}\right\}}}\left|\vec{j}_{2 n}\right\rangle_{a} \otimes\left|\vec{q}_{N-2 n}\right\rangle_{b}, \\
\left|\psi_{L}(N)\right\rangle_{o o} & =\mathcal{N}_{o o, L, N}^{-1 / 2} \sum_{n=0}^{N / 2-1} \sum_{\substack{\left\{j_{2 n+1}\right\},\left\{\hat{q}_{N-2 n-1}\right\}}}\left|\vec{j}_{2 n+1}\right\rangle_{a} \otimes\left|\vec{q}_{N-2 n-1}\right\rangle_{b},
\end{aligned}
$$

where $\quad \mathcal{N}_{e e, L, N}=\sum_{n=0}^{N / 2}\left(\begin{array}{c}L \\ 2 n\end{array}\right)\left(\begin{array}{c}L \\ N-2 n\end{array}\right) ; \quad \mathcal{N}_{o o, L, N}=$ $\sum_{n=0}^{N / 2-1}\left(\begin{array}{c}L \\ 2 n+1\end{array}\right)\left(\begin{array}{c}L \\ N-2 n-1\end{array}\right)$. The states $|\vec{j}\rangle_{a}$ and $|\vec{q}\rangle_{b}$ are simple generalizations of the states $|\vec{j}\rangle$ defined in Eq. (1) to the wire $a$ and $b$, respectively. These describe the cases of even $(e e)$ or odd $(o o)$ particle numbers in each of the wires. For odd $N$, the ground states $\left|\psi_{L}(N)\right\rangle_{e o(o e)}$ with an even (odd) number of particles in either wire take the identical sum structure as above with the normalization $\mathcal{N}_{e e, L, N}$ in both cases. The wave functions [Eq. (4)] are the unique ground states of the model [22]. An interesting interpretation of $\left|\psi_{L}(N)\right\rangle_{\sigma \sigma^{\prime}}$ is in terms of number projection of the ground state of two decoupled even-parity Kitaev chains $|G\rangle=|\psi\rangle_{e}^{a} \otimes|\psi\rangle_{e}^{b}:$

$\left|\psi_{L}(N)\right\rangle_{e e} \propto \hat{P}_{N}|G\rangle ; \quad\left|\psi_{L}(N)\right\rangle_{o o} \propto \hat{P}_{N} \hat{\ell}_{L}^{a \dagger} \hat{\ell}_{L}^{b \dagger}|G\rangle ;$

$\left|\psi_{L}(N)\right\rangle_{o e} \propto \hat{P}_{N} \hat{\ell}_{L}^{a \dagger}|G\rangle ; \quad\left|\psi_{L}(N)\right\rangle_{e o} \propto \hat{P}_{N} \hat{\ell}_{L}^{b \dagger}|G\rangle ;$

where $\hat{\ell}_{L}^{a}$ and $\hat{\ell}_{L}^{b}$ are the zero-energy modes of the decoupled Kitaev wires at half filling. This interpretation provides intuition that the twofold ground-state degeneracy is absent for periodic boundary conditions: since on a circle $\hat{H}_{K}$ has a unique ground state, the ground state of $\hat{H}$ with $N$ particles is also unique [22].

Important evidence of a topologically nontrivial bulk state is obtained from the double degeneracy of the entanglement spectrum, which we now compute for one of the wave functions [Eq. (4)]. To this end, we consider the reduced state of $l$ sites on each wire $\rho_{l}=$ $\operatorname{Tr}_{(L-l)}\left[\left|\psi_{L}(N)\right\rangle_{e e}\left\langle\left.\psi_{L}(N)\right|_{e e}\right]\right.$. Taking the symmetries into account, it can be written in diagonal form as [22]

$$
\rho_{l}=\sum_{N_{l}=0}^{\min (2 l, N)} \sum_{\sigma, \sigma^{\prime}} \chi_{\left(\sigma \sigma^{\prime}, l, N_{l}\right)}^{(L, N)}\left|\psi_{l}\left(N_{l}\right)\right\rangle_{\sigma \sigma^{\prime}}\left\langle\left.\psi_{l}\left(N_{l}\right)\right|_{\sigma \sigma^{\prime}}\right.
$$

with the following nonzero eigenvalues: for $N_{l}$ even $\chi_{\left[e e(o o), l, N_{l}\right]}^{(L, N)}=\mathcal{N}_{e e(o o), l, N_{l}} \mathcal{N}_{e e(o o), L-l, N-N_{l}} / \mathcal{N}_{e e, L, N}$ whereas for $N_{l}$ odd $\chi_{\left(e o, l, N_{l}\right)}^{(L, N)}=\chi_{\left(o e, l, N_{l}\right)}^{(L, N)}=\chi_{\left(e e, l, N_{l}\right)}^{(L, N)}$. In the oddparticle number sector the entanglement spectrum is
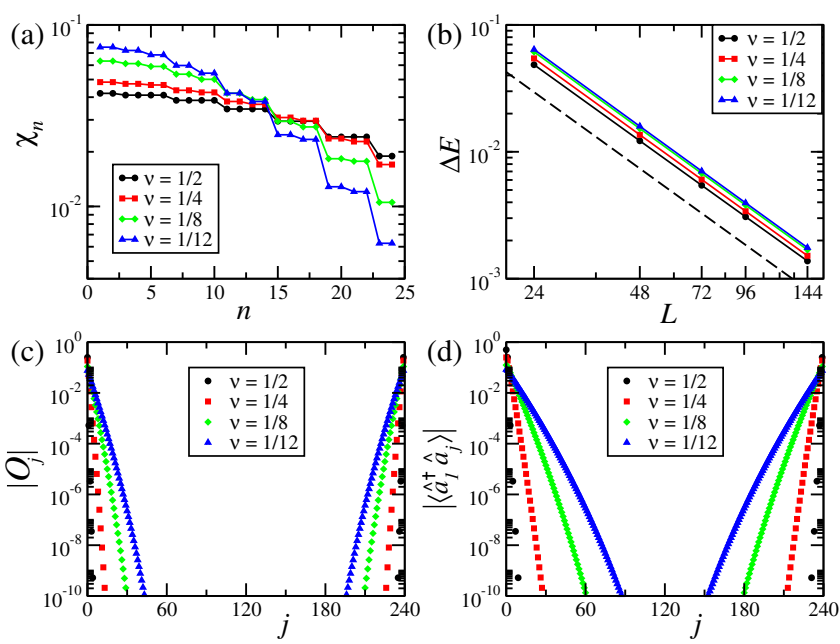

FIG. 2 (color online). Analysis of model $\hat{H}$. (a) Entanglement spectrum for a reduced state $\rho_{l}$ with $l=60$ for $L=240$. (b) DMRG results for the scaling of the gap computed at fixed parity, which is compatible with $L^{-2}$ (dashed line); here the number of kept states is $m=400$. (c) Localization of the edge mode computed via $\left|\left\langle\left.\psi_{L}(N)\right|_{o o} \hat{a}_{j}^{\dagger} \hat{b}_{j} \mid \psi_{L}(N)\right\rangle_{e e}\right|$. (d) Single-fermion edge correlations $\left|\left\langle\hat{a}_{1}^{\dagger} \hat{a}_{j}\right\rangle\right|$ computed for a system of size $L=240$. The wave function is shift invariant, such that $\left|\left\langle\hat{a}_{i}^{\dagger} \hat{a}_{i+j}\right\rangle\right| \equiv\left|\left\langle\hat{a}_{1}^{\dagger} \hat{a}_{j}\right\rangle\right|(i+j \leq L)$.

manifestly twofold degenerate. In the even one, such degeneracy appears in the thermodynamic limit: $\chi_{\left(e e, l, N_{l}\right)}^{(L, N)} / \chi_{\left(o o, l, N_{l}\right)}^{(L, N)} \rightarrow 1$ [see Ref. [22] and Fig. 2(a)].

An interesting insight is provided by $O_{j} \equiv$ $\left\langle\left.\psi_{L}(N)\right|_{o o} \hat{a}_{j}^{\dagger} \hat{b}_{j} \mid \psi_{L}(N)\right\rangle_{e e}$, where $\hat{a}_{j}^{\dagger} \hat{b}_{j}$ is the only singlesite operator which commutes with $\hat{N}$ and changes the local parities $\hat{P}_{a, b}$, so that the two ground states can be locally distinguished. The calculation of such matrix elements leads to a lengthy combinatorial expression [22] and is shown in Fig. 2(c). We interpret the exponential decay of $O_{j}$ into the bulk as a clear signature of localized edge modes with support in this region only. At half filling the edge states are maximally localized, but away from half filling the number projection increases the localization length. In the thermodynamic limit, this length diverges for $\nu \equiv N / 2 L \rightarrow 0,1$, indicating a topological phase transition. We emphasize that this exponential behavior is different from Refs. [10,11], reporting algebraic localization of the edge states, but similar to Refs. $[13,19]$. Nonlocal correlations of edge states are another clear indication of topological order and can be proven via $\left\langle\hat{a}_{1}^{\dagger} \hat{a}_{j}\right\rangle$, which is sizable both at $j \sim 1$ and $j \sim L$ [see the analytical expression in Ref. [22] and Fig. 2(d)].

Furthermore, the Hamiltonian is gapless and hosts long wavelength collective bosonic excitations, while the single fermion excitations experience a finite gap. This is a crucial property of the ground state; the absence of gapless fermion modes in the bulk ensures the robustness of the zero energy edge modes, in analogy to noninteracting topologically 
nontrivial systems. The gapped nature of single fermion excitations is established via the exponential decay of the fermionic two-point function, e.g., $\left\langle\hat{a}_{i}^{\dagger} \hat{a}_{j}\right\rangle$. Again, the resulting formula is a lengthy combinatorial expression [22], evaluated numerically for very large sizes and plotted in Fig. 2(d). For $\nu \rightarrow 0,1$, the correlation length diverges, indicating the vanishing of the fermion gap and a thermodynamic, density-driven phase transition in full analogy to the Kitaev chain.

On the other hand, the analysis of the superfluid correlations demonstrates the existence of gapless modes. The $p$-wave nature of these correlations follows from the correlation of the pairing operator $\hat{a}_{j+1} \hat{a}_{j}$. A direct calculation [22] shows a saturation at large distance

$$
\left\langle\hat{a}_{i}^{\dagger} \hat{a}_{i+1}^{\dagger} \hat{a}_{j+1} \hat{a}_{j}\right\rangle \stackrel{L \rightarrow \infty}{\longrightarrow} \nu^{2}(1-\nu)^{2} .
$$

Similar expressions hold for cross-correlations between the wires. The finite asymptotic value in Eq. (7) hints at the absence of bosonic modes with linear dispersion, which would lead to algebraic decay. A DMRG analysis of the excitation spectrum of $\hat{H}$ for system sizes up to $L=144$ demonstrates a vanishing of its gap $\sim L^{-2}$ [Fig. 2(b)]. This indicates the presence of collective excitations with quadratic dispersion. Further support to this statement is provided from the fact that Eq. (3) without the wire coupling term reduces to the $X X Z$ model at the border of its ferromagnetic phase, which hosts quadratically dispersing spin waves, $\omega \sim q^{2}$. This dispersion, with dynamic exponent $z=2$, gives rise to an effective phase space dimension $d_{\text {eff }}=z+1=3$ at zero temperature, explaining the constancy of superfluid correlations due to the absence of a divergence in the soft mode correlators. This finding is special for $\lambda=1$.

Non-Abelian statistics.-We now proceed to demonstrate that the edge modes obey a non-Abelian statistics completely equivalent to that of Majorana fermions-i.e., Ising anyons. Consider the operator $\hat{B}_{a R, b R}(j)=$ $\left(\hat{\mathcal{I}}+\hat{Z}_{a R, b R, j}\right) / \sqrt{2}$ with $j<L / 2$, where $\hat{Z}_{a R, b R, j}=$ $\left(\sum_{p=1}^{j}\left[\prod_{q=0}^{p-1} \hat{Y}_{a R, b R, q}\right] \hat{X}_{a R, b R, p}\right) / \mathcal{F}(j)$, with $\quad \hat{X}_{a R, b R, j}=$ $\left(a_{L+1-j}^{\dagger} b_{L+1-j}-b_{L+1-j}^{\dagger} a_{L+1-j}\right), \hat{Y}_{a R, b R, j}=n_{L+1-j}^{a} n_{L+1-j}^{b}+$ $\left(1-n_{L+1-j}^{a}\right)\left(1-n_{L+1-j}^{b}\right) \quad$ for $j>0, \quad \hat{Y}_{a R, b R, 0}=\hat{\mathcal{I}} \quad$ and $\mathcal{F}_{j}=\sqrt{1-\left[\nu^{2 j}+(1-\nu)^{2 j}\right]} . \hat{B}_{a R, b R}(j)$ is thus exponentially localized at the right edge of the ladder and an analogous operator $\hat{B}_{a L, b L}(j)$ can be defined at the left edge through the transformation mapping an operator at site $l$ to site $L+1-l$ (and viceversa). Similarly, the operators $\hat{B}_{a R, a L}(j)$ and $\hat{B}_{b L, b R}(j)$ can be defined through the transformations $b_{L+1-l} \rightarrow-i a_{l}$ and $a_{L+1-l} \rightarrow-i b_{l}$, respectively. In general, one can define operators $\hat{B}_{m \Lambda, m^{\prime} \Lambda^{\prime}}(j)$ with $m, m^{\prime}=a, b$ and $\Lambda, \Lambda^{\prime}=L, R$. These operators have the following key properties: They (i) are exponentially localized at the edges, (ii) act unitarily in the ground state subspace, (iii) are particle number conserving, and (iv) most importantly, provide a representation of Majorana braiding operators. From this we conclude that the localized edge modes behave as non-Abelian Majorana fermions [23], in full analogy to the case of two neighboring Kitaev wires. Properties (i)-(iii) are demonstrated in Ref. [22], whereas here we focus on (iv). Strictly speaking, properties (ii) and (iv) are only true apart from an error which is exponentially small in $j$ and $L$, which can always be made negligible. In this case we can define the braiding operator $\hat{R}_{m \Lambda, m^{\prime} \Lambda^{\prime}} \equiv$ $\hat{B}_{m \Lambda, m^{\prime} \Lambda^{\prime}}(j)$. We initialize the system in the state $\left|\psi_{L}(N)\right\rangle_{e e}$ and then perform two braiding operations on the edges in different sequences. If we consider for example $\hat{R}_{a R, a L}$ and $\hat{R}_{a R, b R}$ we obtain $\left[\hat{R}_{a R, a L}, \hat{R}_{a R, b R}\right]\left|\psi_{L}(N)\right\rangle_{e e}=i\left|\psi_{L}(N)\right\rangle_{o o}$ [22] which demonstrates the non-Abelian character of these operations. Moreover, this is the pattern that the conventional braiding operators produce on two neighboring Kitaev wires $\hat{\mathcal{R}}_{m \Lambda, m^{\prime} \Lambda^{\prime}}^{\prime}=e^{\pi / 4 \gamma_{m \Lambda} \gamma_{m^{\prime} \Lambda^{\prime}}}=\left(\mathcal{I}+\gamma_{m \Lambda} \gamma_{m^{\prime} \Lambda^{\prime}}\right) /$ $\sqrt{2}$, where $\gamma_{m \Lambda}$ are Majorana operators fulfilling the Clifford algebra appearing at the left and right $(\Lambda=L, R)$ edges of two Kitaev wires $m=a, b$. This pattern coincides for the application of $\left[\hat{R}_{m \Lambda, m^{\prime} \Lambda^{\prime}}\right.$, $\left.\hat{R}_{n \Upsilon, n^{\prime} \Upsilon^{\prime}}\right]$ on all $\left|\psi_{L}(N)\right\rangle_{\sigma \sigma^{\prime}}$ states (see, e.g., Ref. [24]). In other words, the operators $\hat{R}_{m \Lambda, m^{\prime} \Lambda^{\prime}}$ form a numberconserving representation of Majorana braiding operators on the ground state subspace.

Numerical results. - To further explore the status of these results, we now move to the full model $\hat{H}_{\lambda}$ away from the solvable line $\lambda=1$. The study is performed with the DMRG on systems with sizes up to $L=240$ and open boundary conditions.

We first establish the absence of a topological phase for $\lambda>1$. The density profile, shown in the inset of Fig. 1 for $\nu=0.5$ and $\lambda=1.01$, displays a clear phase-separation tendency. Analogous data are obtained for other values of $\nu$ (see dark crosses in Fig. 1). These results can be intuitively understood considering that $\hat{H}_{\lambda>1}$ without interwire coupling can be mapped to a gapped ferromagnetic $X X Z$ model with domain walls dual to fermionic phase separation.

For $\lambda<1$, simulations support the existence of a homogeneous phase (Fig. 1). Note that $\lambda=0$ is a freefermion point trivially nontopological. For $\lambda \neq 0$ we observe (i) two quasidegenerate ground states with different relative parity and same particle numbers, (ii) a degenerate entanglement spectrum, (iii) a gap closing as $L^{-1}$ for fixed parity, (iv) exponentially decaying single-fermion correlations, and (v) power-law decaying superfluid correlators. Plots in Fig. 3 display our numerical results. Simulations at lower filling $\nu \rightarrow 0$ and small $\lambda$ are more demanding, owing to the increasing correlation length of the system. The numerics is consistent with the phase diagram in Fig. 1 exhibiting a topological phase delimited by three trivial lines at $\lambda=0, \nu=0$ and $\nu=1$ and an inhomogeneous nontopological phase for $\lambda>1$. The exactly solvable 

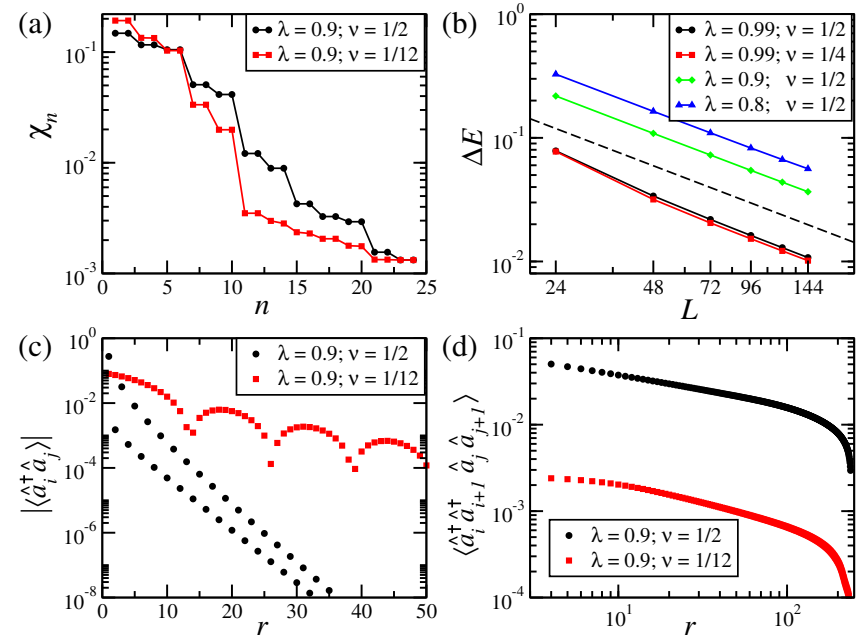

FIG. 3 (color online). DMRG results for model $\hat{H}_{\lambda}$. (a) Entanglement spectrum for a reduced state $\rho_{l}$ with $l=100$ for $L=240$ ( $m=300)$. (b) Algebraic scaling of the gap computed at fixed parity, which is compatible with $L^{-1}$ (dashed line). Here $m=420$. (c),(d) Single-particle $\left|\left\langle\hat{a}_{i}^{\dagger} \hat{a}_{j}\right\rangle\right|$ and $p$-wave superfluid $\left\langle\hat{a}_{i}^{\dagger} \hat{a}_{i+1}^{\dagger} \hat{a}_{j} \hat{a}_{j+1}\right\rangle$ correlations at distance $r=|i-j|$ computed in the bulk of system with $L=240(m=300)$. Analogous data were obtained for other values of $\nu, \lambda$ (red circles in Fig. 1).

topological line at $\lambda=1$ serves as a boundary; the fixed- $\nu$ phase diagram is reminiscent of the ferromagnetic transition in the $X X Z$ model.

Conclusions. - We presented an exactly solvable twowire fermionic model which conserves the number of particles and features Majorana-like exotic quasiparticles at the edges. Our results can be a valuable guideline to understand topological edge states in number conserving systems. For example, the replacement $\hat{a}_{i} \rightarrow \hat{c}_{i, \uparrow}, \hat{b}_{i} \rightarrow \hat{c}_{i, \downarrow}$ results in a one-dimensional spinful Hubbard Hamiltonian without continuous spin rotation, but time reversal symmetry. The resulting model with an exactly solvable line belongs to the class of time reversal invariant topological superconductors [25], analyzed in a number conserving setting recently [19], with edge modes protected by the latter symmetry. Moreover, exactly solvable number conserving models can be constructed in arbitrary dimension.

We thank E. Altman, M. Baranov, E. Berg, J. C. Budich, M. Burrello, M. Dalmonte M Herrmanns, and G. Ortiz for enlightening discussions. F. I. acknowledges financial support by the Brazilian agencies FAPEMIG, CNPq, and INCT- IQ (National Institute of Science and Technology for Quantum Information). S. D. acknowledges support via the START Grant No. Y 581-N16 and the German Research Foundation through ZUK 64. R. F. acknowledges financial support from the EU integrated project SIQS and from Italian MIUR via PRIN Project No. 2010LLKJBX. D. R. and L. M. acknowledge the Italian MIUR through FIRB Project No. RBFR12NLNA. L. M. was supported by Regione Toscana POR FSE 2007-2013.
Note added.-Recently, we became aware of similar results obtained by Lang and Büchler [26].

[1] M. Z. Hasan and C. L. Kane, Rev. Mod. Phys. 82, 3045 (2010).

[2] X.-L. Qi and S.-C. Zhang, Rev. Mod. Phys. 83, 1057 (2011).

[3] C. Nayak, S. H. Simon, A. Stern, M. Freedman, and S. Das Sarma, Rev. Mod. Phys. 80, 1083 (2008).

[4] A. Y. Kitaev, Phys. Usp. 44, 131 (2001).

[5] R. M. Lutchyn, J. D. Sau, and S. Das Sarma, Phys. Rev. Lett. 105, 077001 (2010).

[6] Y. Oreg, G. Refael, and F. von Oppen, Phys. Rev. Lett. 105, 177002 (2010).

[7] M. Sato, Y. Takahashi, and S. Fujimoto, Phys. Rev. Lett. 103, 020401 (2009).

[8] L Jiang, T. Kitagawa, J. Alicea, A. R. Akhmerov, D. Pekker, G. Refael, J. I. Cirac, E. Demler, M. D. Lukin, and P. Zoller, Phys. Rev. Lett. 106, 220402 (2011).

[9] V. Mourik, K. Zuo, S. M. Frolov, S. R. Plissard, E. P. A. M. Bakkers, and L.P. Kouwenhoven, Science 336, 1003 (2012).

[10] L. Fidkowski, R. M. Lutchyn, C. Nayak, and M. P. A. Fisher, Phys. Rev. B 84, 195436 (2011).

[11] J. D. Sau, B. I. Halperin, K. Flensberg, and S. Das Sarma, Phys. Rev. B 84, 144509 (2011).

[12] M. Cheng and H.-H. Tu, Phys. Rev. B 84, 094503 (2011).

[13] C. V. Kraus, M. Dalmonte, M. A. Baranov, A. M. Läuchli, and P. Zoller, Phys. Rev. Lett. 111, 173004 (2013).

[14] G. Ortiz, J. Dukelsky, E. Cobanera, C. Esebbag, and C. Beenakker, Phys. Rev. Lett. 113, 267002 (2014).

[15] S. Diehl, W. Yi, A. J. Daley, and P. Zoller, Phys. Rev. Lett. 105, 227001 (2010).

[16] S. Diehl, E. Rico, M. A. Baranov, and P. Zoller, Nat. Phys. 7, 971 (2011).

[17] C.-E. Bardyn, M. A. Baranov, C. V. Kraus, E. Rico, A. Imamoglu, P. Zoller, and S. Diehl, New J. Phys. 15, 085001 (2013).

[18] J. Ruhman, E. Berg, and E. Altman, Phys. Rev. Lett. 114, 100401 (2015).

[19] A. Keselman and E. Berg, Phys. Rev. B 91, 235309 (2015).

[20] A. M. Turner, F. Pollmann, and E. Berg, Phys. Rev. B 83, 075102 (2011).

[21] S. Sachdev, Quantum Phase Transitions (Cambridge University Press, Cambridge, England, 1999).

[22] See Supplemental Material at http://link.aps.org/ supplemental/10.1103/PhysRevLett.115.156402 for additional information on the analytical derivations of the exact results presented in the Letter.

[23] A. Yu Kitaev, Ann. Phys. (Amsterdam) 321, 2 (2006).

[24] C. Laflamme, M. A. Baranov, P. Zoller, and C. V. Kraus, Phys. Rev. A 89, 022319 (2014).

[25] X.-L. Qi, T. L. Hughes, S. Raghu, and S.-C. Zhang, Phys. Rev. Lett. 102, 187001 (2009).

[26] N. Lang and H. P. Büchler, Phys. Rev. B 92, 041118 (2015). 